\title{
An appreciation of Dr. Stephen M. Haley, PT, PhD, FAPTA
}

\author{
Alan M. Jette
}

Published online: 5 October 2011

(C) Springer Science+Business Media B.V. 2011

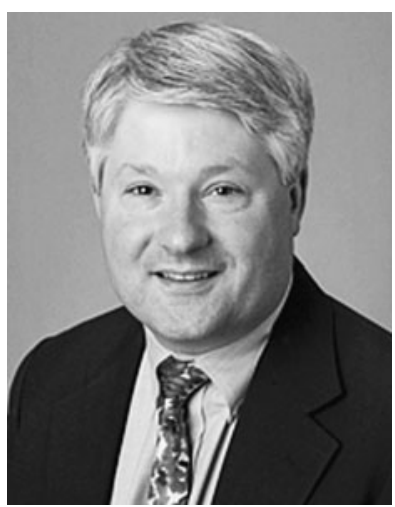

Stephen M. Haley, Professor of Health Policy and Management and Associate Director of the Health and Disability Research Institute at Boston University's School of Public Health, died on July 16, 2011 after a long and debilitating illness. Dr. Haley had a remarkable career as a pediatric physical therapist, researcher, educator and mentor.

A native of Ohio, Dr. Haley received his BS in Psychology and Certificate in Physical Therapy from Ohio State University, becoming a fervent and life-long Buckeyes football fan. He received a MS in Education from the University of Kentucky and a PhD in Educational Psychology from the University of Washington.

In the late 1980s and early 1990s, Dr. Haley was a researcher in the Medical Rehabilitation Research and Training Center on Rehabilitation and Childhood Trauma and was Research Associate Professor in the Department of

\footnotetext{
A. M. Jette $(\bowtie)$

Health and Disability Research Institute, Boston University

School of Public Health, Boston, MA, USA

e-mail: ajette@bu.edu
}

Rehabilitation Medicine at Tufts University School of Medicine. There he led a team that developed the Pediatric Evaluation of Disability Inventory (PEDI), a clinical and research assessment instrument, first published in 1991, that has become a preferred measure that is used worldwide to evaluate the functioning of children with disabilities. Prior to the PEDI, most pediatric assessments were guided by a developmental model that focused on the extent to which children with disabilities do or do not attain motor, cognitive, and social developmental milestones at the expected age. The PEDI changed pediatric measurement by providing rehabilitation specialists a way to measure functional abilities in children and to track their progress over time. An innovation of the original PEDI was the use of Rasch item response theory methods for item scaling that eventually became the standard for other pediatric outcome measures. From 2004 to 2009, Dr. Haley was awarded a prestigious K02 Independent Scientist Award from the NCMRR/NIH, to develop his capacity further to design and deliver computer adaptive test applications for the assessment of functional status. During this period, Dr. Haley and colleagues applied these methodologies to create a CAT version of the PEDI instrument which is being disseminated worldwide. This newer PEDI-CAT provides rehabilitation professionals a more efficient way of assessing functional abilities in children and youth from birth to 21 years.

As Research Associate at The Health Institute at New England Medical Center in 1992-1993, Dr. Haley led a team of researchers that published groundbreaking articles examining the hierarchical structure, unidimensionality and reproducibility of items included within the SF-36 health related quality of life scales, using Rasch Item Theory Response methodology. This methodology has since become the standard in the rehabilitation field for constructing and evaluating health outcome instruments. 
From 1999 to 2004, as Director of Research in the Rehabilitation Research and Training Center for Measuring Rehabilitation Outcomes at Boston University, Dr. Haley and colleagues pioneered the innovative combination of IRT and computer-adaptive test (CAT) methodologies to create the Activity Measure for Post-Acute Care (AM-PAC). The AM-PAC CAT instrument, which has been translated in over 20 languages, is the first functional outcome instrument designed to assess patient outcomes across an entire episode of post-acute care, regardless of the setting where care is received. Using the AM-PAC, Dr. Haley and colleagues published innovative research illustrating how IRT methods could be employed to replenish CAT assessment instruments and how to create functional stages to assist in the interpretation of quantitative outcome scores.

In 1998, Dr Haley began serving as the Director of Research at Franciscan Hospital for Children, a pediatric rehabilitation hospital located in Boston, MA. Dr Haley hoped to link the efforts and expertise of academic researchers with clinicians to promote advances in rehabilitative care for children with disabilities. For 13 years, Dr Haley guided clinicians and researchers in developing competitive grant proposals to support the work of Franciscan's Research Center for Children with Special health Care Needs. He worked with staff to develop intervention studies in the areas of physical fitness and aquatic physical therapy and to develop outcome studies in the area of inpatient rehabilitation, primarily for children with brain injuries. He instructed the hospital staff in effective ways to measure outcomes for individual patients as well as for overall hospital programs and was instrumental in the creation of the Specialized Pediatric Applied Research Collaborative (SPARC), a multisite clinical research consortium of post-acute pediatric rehabilitation hospitals created to respond to the need to establish clinical outcome data for the development of program standards; monitor and improve quality of care; and communicate realistic expectations to children and families.

Over a decade, Dr. Haley worked with colleagues at the Shriners Hospitals for Children on developing and testing CAT platforms for physical functioning, activity and participation. As a result of this collaboration, 10 large item banks have been developed and calibrated on children and parents with cerebral palsy, spinal cord injury and birth brachial plexus injuries. At the time of Dr. Haley's death, work was just completed on calibrating the items on over 2,800 typically developing children and their parents. The influence of Dr. Haley's work on the Shriners Hospitals for Children is only in its infancy. His legacy will live-on permanently as the studies he co-led will continue to completion and new studies which he helped design will be implemented. Collectively, Dr. Haley's work will create a complete transformation in the method in which the
Shriners Organization will be able to monitor outcomes across their 22 hospitals and will provide Shriners and other pediatric health care centers with a robust and efficient system to measure important outcomes from the perspectives of children and their parents.

As Dr. Mulcahey, Director Rehabilitation Services and Clinical Research at Shriners Hospitals For Children noted, "While Steve's intellectual and academic contributions to Shriners Hospitals for Children have been pivotal and transformational, it was Steve's unique personality, unforgettable wit, unassuming sarcasm and love for meaningful relationships that will forever be remembered." Dr. Dave Cella, Professor and Chair of the Department of Medical Social Sciences, Northwestern University Feinberg School of Medicine, summarized Dr. Haley's impact on the field of outcome measurement as follows: "The past two decades have been transformative in the patient-reported outcomes (PRO) measurement field. The two most important advances in PRO measurement since 1990 have been the introduction and application of item response theory into health measurement, and the production of accessible, interpretable instrumentation for the end-user who doesn't specialize in PRO measurement. Steve Haley has been a true leader in both of these areas. Always ahead of the pack, Steve was writing about IRT measurement and developing practical applications for rehabilitation settings in both pediatric and adult settings. Steve was modest about his accomplishments. He was not a showman, but those of us who read his work saw that he was out in front of so many others, scientifically and practically. Conversations with Steve never went very far before he was making it clear that his passion about helping people make the best of their lives as they live with chronic illness or disability. Steve did the very same thing with his own illness."

Throughout his career, Dr. Haley was a teacher and mentor to many doctoral, postdoctoral and junior investigators. He was a gifted speaker and highly skilled teacher who generously shared his knowledge with his students. His passion for improving the lives of children with disabilities by improving the quality of rehabilitation research was an inspiration to the students and mentees who worked with him. Dr. Haley received numerous awards for his research and scholarship including, in 1993, the Golden Pen Award for contributions to the Physical Therapy Journal and, in 2006, the Helen Hislop Award for Outstanding Contributions to the Professional Literature in Physical Therapy. In 2009, he was elected as a Catherine Worthingham Fellow of the American Physical Therapy Association. Dr. Haley was a world renowned innovator in pediatric and adult outcome measurement. As Dr. Scott Ward, President of the American Physical Therapy Profession, affirmed, "Our profession has lost a great thinker, mentor, pioneer and friend." 